\title{
Maternal Physiological Changes in Pregnancyand Pathophysiology of COVID-19
}

Jan Pawel Andrade Pachnicki ${ }^{1,2,3,4^{*}}$, Liz Ribeiro Wallim ${ }^{2 \dagger}$, Gabriela Vanim de Moraes $^{1 \dagger}$ and Sarah Oliveira de Lima ${ }^{1+}$

*Correspondence: janpawel@uol.com.br

'These authors contributed equally to this work.

${ }^{1}$ Positivo University, Curitiba, Brazil.

${ }^{2}$ Pontifical Catholic University of Paraná, Curitiba, Brazil.

${ }^{3}$ Federal University of Paraná, Curitiba, Brazil.

${ }^{4}$ Mackenzie Evangelical College of Paraná, Curitiba, Brazil.

\begin{abstract}
Based on previous knowledge of other epidemics such as SARS, MERS and H1N1, it is proposed that pregnant women may have a severe clinical outcome in some situations. It is not different for COVID-19. The control of SARS-CoV-2 infection among pregnant women and their potential risk of vertical transmission are a concern to the medical and scientific community. However, the maternal organism does not behave the same way during those forty weeks. Here we show the differences in pathophysiology of COVID-19 in pregnant women as a consequence of physiological and immunological changes related to each gestational period, in order to guide an appropriate therapy and care for this population. We observed that these women are in a transient physiological condition in which ACE-2 is highly expressed and, despite its protective mechanism for pregnancy, it is also used by the virus as a route of entry. Moreover, their physiological immunosuppression associated with cytokines increased levels, may lead to a cytokine storm, especially in the first trimester and at the end of pregnancy (pro-inflammatory stages). Therefore, we found in this review that individualization of each gestational period should be practiced not considering pregnancy as a unique status.
\end{abstract}

Keywords: Maternal Physiological Changes, Pathophysiology, COVID-19

\section{Introduction}

The COVID-19 (COronaVIrusDisease 2019) pandemic, caused by the new coronavirus, spreads rapidly across all continents, becoming a global threat. Since its emergence in December 2019 in the city of Wuhan, China [1], the virus has been causing great concern to public health authorities [2], leading the World Health Organization (WHO) to declare a COVID-19 pandemic on March 11, 2020. According to the WHO, more than five million cases have been confirmed worldwide, leading to more than 360,000 deaths by May 30, 2020 [3].

As a consequence of physiological and immunological changes related to the gestational period, it seems that pregnant women are more susceptible to respiratory pathogens, although there is still no confirmation on the hypothesis that clinical manifestations of COVID-19 in pregnant women are different from non-pregnant ones [4,5]. However, in previous pandemics, such as SARS, MERS and H1N1, pregnant women were notoriously more susceptible to severe diseases, causing a higher morbidity and mortality rate than general population [6].

Considering the data already obtained regarding this population group, the control of infection among pregnant women and their potential risk of vertical transmission are a concern to the medical and scientific community [7], once in this period, there is an increase in the expression of the ACE-2 enzyme, used as pathogen's entrance route. In addition, the natural immunological process in pregnancy provides an immunomodulation state, possibly capable to promote a deregulated response when fightingagainst the virus [8].

\section{Review \\ COVID-19 in Pregnancy}

The disease is normally characterized by infection of the superior respiratory tract with fever and cough, in addition to infection of the lower respiratory tract varying mild to severe, which can lead 
Pachnicki et al, Archives of Physiology 2021,

http://www.hoajonline.com/journals/pdf/2055-0898-8-1.pdf

doi: $10.7243 / 2055-0898-8-1$

to severe acute respiratory syndrome (SARS) [9]. Given an R0 (transmission rate) higher than that observed in other epidemics caused by coronavirus or influenza viruses, COVID-19 quickly reached the five continents through personal contact, sneezing, coughing or through secretions of infected people [10].

As pandemic extends, infection control among pregnant women and their potential risk of vertical transmission becomes a major concern [7]. When observing previous epidemics of emerging viral infections, poor obstetric outcomes were found, such as maternal-fetal transmission of the virus, perinatal infections and maternal and neonatal mortality. Thus, it is perceived that the common physiological and immunological changes in pregnancy are capable of increasing the risk of complications from respiratory infections. Changes in the cardiovascular and respiratory system, such as an increase in heart rate, circulating blood volume and oxygen consumption, combined with a decrease in lung capacity, increase the risk of developing a serious respiratory disease. In addition, there are still immunological adaptations that allow pregnant women to tolerate a genetically distinct fetus [11], reinforcing the fact that pregnant women should be treated as a risk group.

A study of 118 infected women in Wuhan showed there is not enough data to prove a higher risk of severe disease in pregnant women, as occurred with influenza. In this article, about $92 \%$ of pregnant women presented mild disease and there were no cases of vertical transmission. The main symptoms identified were fever, cough, and lymphocytopenia [12]. According to previous data on SARS and MERS, clinical findings may change from absence of symptoms to severe symptoms, and death, during pregnancy [13].

In a recent study based on the description of 43 cases of COVID-19 confirmed in pregnant women in New York City hospitals, fourteen (32.6\%) patients had no viral symptoms associated with SARS-CoV-2, that were only identified after the development of these symptoms during admission or after the universal test for all obstetric admissions. Among these, 10/14 (71.4\%) developed symptoms of COVID-19 during admission until the time of delivery or soon after postpartum discharge. Of the remaining 29 (67.4\%) patients who presented symptomatic infection by SARS-CoV-2, three required prenatal admission due to infection symptoms, and an additional patient presented deterioration of respiratory status, requiring oxygen supplementation [6]. In another study performed by Wu et al, 37 (86\%) women had mild disease, four $(9.3 \%)$ had severe disease and two (4.7\%) had critical disease; these percentages are similar to those described for non-pregnant women with COVID-19 (about $80 \%$ of mild disease, $15 \%$ severe and $5 \%$ critical). However, the data are still limited and insufficient, leading to doubts regarding the presentation of more severe symptoms in pregnant women [2].

\section{Viral morphology and invasion mechanism into the host cell}

Coronaviruses are a group of single stranded, non-segmented, enveloped RNA genome viruses capable of promoting disorders ranging from common colds to severe, fatal diseases. Before the COVID-19 pandemic, only two types of coronaviruses already known led to severe respiratory diseases in humans: SARS-CoV and MERS-CoV. With the emergence of SARS-CoV-2, a third coronavirus showed capability of causing severe respiratory disease, even resulting in more cases than SARS and MERS together [14].

A study published in February 2020 showed that SARS-CoV and SARS-CoV-2 share, on average, $75 \%$ to $80 \%$ of the same genome sequence, revealing countless similarities between these two viruses [15].

Nasopharyngeal and oropharyngeal isolated samples of SARS-CoV-2 were inoculated in order to perform cell culture of this pathogen. Thus, it was discovered that the structure of the new coronavirus consists in particles ranging from 70 to $90 \mathrm{~nm}$, with numerous cytoplasmic organelles specifically surrounded by vesicles [16]. In addition, SARS-CoV-2 has, in its structure, surface proteins like the Spike protein (S), membrane protein $M$, and small membrane protein $E$ [17]. Viral entry occurs via two routes. The first occurs when the Spike protein attaches to the angiotensin-converting enzyme 2 (ACE-2) receptor, releasing the viral genome and nucleocapsid protein into the host cell cytoplasm. The other pathway is the direct plasma membrane route via transmembrane serine protease 2, which allows for the proteolytic cleavage of the spike protein and mediation of fusion with the cell membrane. Intracellularly, the viral genome is translated into a replicase to produce more genome RNA, mRNA and viral protein [18].

Both SARS-CoV and SARS-CoV-2 have similarity regarding the viral invasion into the target cell, taking advantage of ACE-2 for this purpose [19]. Thus, the binding between Spike protein and ACE- 2 receptor promotes a conformational modification that allows virus' entrance into the cell through the fusion of the viral envelope with the host cell membrane [20]. However, studies have shown a crucial difference between SARS-CoV and SARS-CoV-2: the presence of Furin. This enzyme is not activated during the host cell invasion process in SARS-CoV and MERS-CoV infection, what denotes that its presence may be related to the severity and/or transmission facility of COVID-19 [21].

SARS-CoV-2 has a Furin cleavage site that is located at the Spike protein. This type of cleavage site is usually found in highly virulent forms of the influenza virus, which help in the expansion of virus' cellular tropism [22]. Therefore, it is believed that Furin, when cleaved, assists in SARS-CoV-2 cellular tropism. Several human organs expresses it, such as lungs, small intestine and liver, what proves the reason why the new coronavirus is extremely contagious in conjunction with its potential ability to infect multiple organs and the genetic differences in immune response between hosts [21].

\section{Expression and activity of ACE-2 during pregnancy} Li et al, in 2003, identified that protein ACE-2 (a metallo- 
peptidase responsible for converting Angiotensin I into Ang 1-9 and Angiotensin II into Ang 1-7, with vasodilatory, antithrombotic, and anti-inflammatory activities), target of virus Spike protein, is the receptor used by SARS- CoV to enter the cell [23]. This binding is capable to melt the virus membrane to the host cells and to internalize it down regulating the ACE-2 expression [24].

It was observed that both SARS-CoV and SARS-CoV-2 share the same binding locus of the Spike protein: the ACE-2 receptor of the host cell. In addition, studies have shown that the Spike protein in SARS-CoV-2 expresses an affinity 10 to 20 times higher for ACE-2 than SARS-CoV [19], what may explains the high transmission rates.

ACE-2 plays a central role in the non-classic renin-angiotensin-aldosterone system (RAAS), which activity seems to be balanced by the classical pathways under normal physiological conditions. However, it has been found that the new coronavirus, through the activity in the non-classic renin-angiotensin-aldosterone pathway, is capable of causing lung injury and inflammation [23]. Although the virus infects the respiratory epithelium, this enzyme may only be an entry route, since that endothelial lesions resulting in the production of cytokines and recruitment of innate and adaptive cells can mediate the stages of alveolar damage. The tissue tropism of SARS-Cov-2 is related to the expression of ACE-2, which includes lung, gastrointestinal tract and liver. It was seen that in humans, ACE-2 protein is also present in alveolar epithelial cells, serous and bronchial glands, trachea, bronchi, and alveolar pneumocytes and macrophages [25].

Furthermore, Levy et al. investigated the expression and activity of ACE-2 during pregnancy in normotensive and hypertensive rats. It was confirmed that reproductive organs, such as placenta and uterus, in addition to the kidneys, contribute significantly to the expression of ACE-2, leading to the conclusion that pregnancy is a temporary physiological condition in which ACE- 2 is over-expressed and active. It was also shown that increased ACE- 2 expression contribute to decrease vascular resistance and elevate ANG II levels during pregnancy. Therefore, the hypothesis is that these components, ACE-2 and ANG 1-7, would prevent the blood flow disruption between uterus and placenta, the development of systemic arterial hypertension, and could explain the blood pressure reduction during the gestational period [26-28]. ACE-2 is important for the normal development of pregnancy in normotensive and hypertensive individuals and changes in its expression may lead to abnormalities in the blood pressure pattern during pregnancy [26].

This report raises the hypothesis that pregnant women would be more susceptible to the virus (mediated by greater viral replication due to increased receptors at this stage), in addition to explaining some clinical manifestations similar to Preeclampsia that occur in this population when affected by COVID-19. However, based on data from previous coronaviruses infections (MERS and SARS), there is no evidence yet to prove that pregnant women are more susceptible to these pathogens. By the way, since the outbreak of the new coronavirus infection, more men have been affected than women [14].

\section{Deployment of the inflammatory response at COVID-19 and aspects of pregnant women immune system}

The two types of coronavirus previously known to trigger severe pneumonia, SARS-CoV and MERS-CoV, exhibit this clinical manifestation associated with a fast viral replication increasing pro-inflammatory cytokines. Cytokines are well known for playing an important role in the immune response, but at exacerbated levels they can promote an unregulated response [8]. The cytokine storm involves an excessive and damaging immune system response to the pathogen, "reversing" the cytokines' major characteristic - being the chemical molecules that signalize and guide an effectively beneficial immune response. Hence, during a cytokine storm, the levels of these molecules are overly elevated what causes an attack on healthy tissues [29].

Similarly, SARS was also characterized by an exacerbated inflammatory response leading to pulmonary damage. A previous study in mice demonstrated that SARS has a fast viral replication combined with a delayed type 1 interferon (IFN-I) signaling, promoting an accumulation of monocyte and macrophage inflammation. Consequently, it generates high levels of lung's cytokines and chemokines and abnormal T cell responses [8]. Chen et al. [30] made it possible to take a new look at COVID-19 by following contaminated patients during a period of 7 to 10 days in which the cytokine storm occurred at its maximum expression at the peak of respiratory discomfort [8].

Therefore, SARS-CoV-2 infection causes cytokine storm and lymphopenia through a decrease in TCD4+ and TCD8+ cells, in addition to suppression of IFN- $\gamma$ by the TCD4+ cells themselves. Moreover, the concentrations of pro-inflammatory and anti-inflammatory cytokines - including IL-2R, IL-6, TNF- $a$ and IL-10 - increased in severe cases, suggests an association with the severity of COVID-19. The cellular origin and the mechanisms involved in the huge increase of cytokine levels in COVID-19 deserve to be further explored in order to develop the ideal clinical management of this disease [30].

The gestational period comprises a peculiar phase in concern to the immune system that may lead to diseases exacerbation [31]. Progesterone is known as an inducer of Th2-type cytokines, such as interleukin 3 (IL-3), IL-4 and IL-10 [32].

So far, there are two theories that try to justify the gestational phenomenon of relative immunosuppression. One of them points out that this scenario is an immunological adaptation with the purpose of protecting the fetus. The second idea defends the importance of the maternal immune response in relation to the paternal antigens present in the fetus [31]. First, the role of progesterone must be highlighted, since, besides maintaining an appropriate environment for pregnancy, it 
Pachnicki et al, Archives of Physiology 2021,

promotes an immunologically favorable environment for fetal development. According to the "Th1/Th2 paradigm", Th2 cytokines, found at the fetal maternal interface, appear to be related to successful pregnancy outcomes, while Th1 cytokines, such as Interferon gamma (IFN- $\gamma$ ), tumor necrosis factor (TNF), IL-1 and IL-2, are related - when in overproduction - to pregnancy loss.

Therefore, it is normal to observe a relative suppression of Th1-type cytokines, causing a tendency to Th2-cytokines response during uneventful pregnancy, and followed by an increased response in favor of anti-inflammatory cytokines [31]. Thus, pregnancy is divided into three periods by the inflammatory stage: the first trimester, which occurs a proinflammatory stage due to the implantation process, the second anti-inflammatory stage related to fetal growth, and finally the late third trimester, in which a second pro-inflammatory stage occurs indicating the beginning of labor, with the decline of progesterone [33]. This fact characterizes the importance of proper management of this population group, since the same infection will have different inflammatory developments in different gestational periods.

In relation to anti-inflammatory cytokines, the role of IL-10 in the gestational period is also highlighted. The IL-10 is capable of acting in an autocrine and paracrine mode. This occurs in response to inflammation signs of the immune system, enclosing these inflamed sites [34]. This cytokine is highly important during pregnancy since it suppresses the production of pro-inflammatory cascades by other cells, with numerous studies proving its origin at the maternal-fetal interface [35]. Other interesting factors regarding the immunological adaptation to the fetus are seen. For example, the increased levels of estrogen and progesterone in the upper respiratory tract associated with a restriction of lung expansion, which combined promotes the susceptibility of pregnant women to contract respiratory pathogens [36].

There are many evidences that systemic viral infections affect pregnancy and turns the risk greater than in non-pregnant women, with higher morbidity and mortality [37]. An example of this rigorous response to viral crisis is SARS, which during pregnancy has shown high incidences of miscarriages in the first period, in addition to premature births and limited fetal growth in the third period [38].

Regarding interleukin 6 specifically, it is responsible for commanding the expression of C-reactive protein (CRP), which is a biological marker related to cases of severe COVID-19 manifestations [39]. In addition, studies have shown the link between IL- 6 and CRP high levels in amniotic fluid and an increased risk of spontaneous premature delivery in asymptomatic women [40], directly correlated to severe COVID-19.

Thereby, physicians cannot ignore the potential risk that serious cases of COVID-19 may cause in pregnant women. Based on the knowledge that in the first trimester and near the term these patients are in a pro-inflammatory state, if they are infected with SARS-Cov-2, an induced cytokines storm may occur resulting in severe inflammation [36].

\section{Conclusion}

So far, there is not enough data regarding the effect of the new coronavirus on pregnant women. However, based on previous knowledge of other epidemics such as SARS, MERS and $\mathrm{H} 1 \mathrm{~N} 1$, it is proposed that pregnant women may have a severe clinical outcome in some situations. Several factors may categorize these patients as a risk group. First, the fact that these women are in atransient physiological condition in which ACE- 2 is highly expressed and, despite its protective mechanism for pregnancy, it is also used by the virus as a route of entry. Moreover, because they have a physiological immunosuppression associated with cytokines increased levels, the occurrence of a severe infection is capable of generating a cytokine storm, especially in the first trimester and at the end of pregnancy (pro-inflammatory stages). Finally, it is worth mentioning that the normal patterns of maternal physiological changes also corroborate the susceptibility of pregnant women to respiratory infections. Therefore, to adequate prevention and treatment strategies, more evidence about this new disease is needed in this population group, especially in high-risk pregnant women.

\section{Competing interests}

The authors declare that they have no competing interests.

Authors' contributions

\begin{tabular}{|l|c|c|c|c|}
\hline Authors' contributions & JAP & LRW & GVM & SOL \\
\hline Research concept and design & $\checkmark$ & $\checkmark$ & -- & -- \\
\hline Collection and/or assembly of data & $\checkmark$ & $\checkmark$ & $\checkmark$ & $\checkmark$ \\
\hline Data analysis and interpretation & $\checkmark$ & $\checkmark$ & $\checkmark$ & $\checkmark$ \\
\hline Writing the article & $\checkmark$ & $\checkmark$ & $\checkmark$ & $\checkmark$ \\
\hline Critical revision of the article & $\checkmark$ & $\checkmark$ & -- & -- \\
\hline Final approval of article & $\checkmark$ & $\checkmark$ & $\checkmark$ & $\checkmark$ \\
\hline Statistical analysis & $\checkmark$ & $\checkmark$ & $\checkmark$ & $\checkmark$ \\
\hline
\end{tabular}

\section{Acknowledgement}

We sincerely apologize to all authors whose work could not be cited owing to space constraints.

\section{Publication history}

Senior Editor: David A. Tulis, East Carolina University, USA.

Received: 03-Dec-2020 Final Revised: 18-Jan-2021

Accepted: 22-Jan-2021 Published: 07-Feb-2021

\section{References}

1. Huang $C$, Wang $Y$, Li $X$, et al. Clinical features of patients infected with 2019 novel coronavirus in Wuhan, China. Lancet. 2020;395(10223):497506.

2. Wu F, Zhao S, Yu B, et al. A new coronavirus associated with human respiratory disease in China. Nature 579, 265-269 (2020).

3. World Health Organization. Coronavirus disease 2019 (COVID-19) situation report-131. World Health Organization. 2020. https://www. who.int/docs/default-source/coronaviruse/situation-reports/20200530covid-19-sitrep-131.pdf?sfvrsn=d31ba4b3_2 . Acessed 30 May 2020.

4. Liu D, Li L, Zheng D, et al. Pregnancy and Perinatal Outcomes. Am. J. 
Pachnicki et al, Archives of Physiology 2021,

Roentgenol. 215. 2020 (July):1-6.

5. Yan J, Guo J, Fan C, et al. Coronavirus disease 2019 (COVID-19) in pregnant women: a report based on $\mathbf{1 1 6}$ cases. Am J Obstet. Gynecol. April 2020; XX:x.ex-x.ex.

6. Breslin N, Baptiste C, Gyamfi-Bannerman C, et al. Coronavirus disease 2019 infection among asymptomatic and symptomatic pregnant women: two weeks of confirmed presentations to an affiliated pair of New York City hospitals. Am J Obstet Gynecol MFM [Internet]. 2020;100118.

7. J. Qiao. What are the risks of COVID-19 infection in pregnant women? Lancet, 395 (2020), pp. 760-762.

8. Pedersen SF, Ho YC. SARS-CoV-2: A storm is raging. J Clin Invest. 2020 May 1;130(5):2202-2205.

9. Guan W, Ni Z, Hu Y, et al. Clinical characteristics of 2019 novel coronavirus infection in China. N Engl J Med. 2020 Apr 30; 382:17081720.

10. Heymann DL, Shindo N; WHO Scientific and Technical Advisory Group for Infectious Hazards. COVID-19: what is next for public health? Lancet. 2020;395(10224):542-545.

11. Schwartz DA. An Analysis of 38 Pregnant Women with COVID-19, Their Newborn Infants, and Maternal-Fetal Transmission of SARS-CoV-2: Maternal Coronavirus Infections and Pregnancy Outcomes [published online ahead of print, 2020 Mar 17]. Arch Pathol Lab Med. 2020;10.5858/ arpa.2020-0901-SA.

12. Chen L, Li Q, Zheng D, et al. Clinical Characteristics of Pregnant Women with Covid-19 in Wuhan, China [published online ahead of print, 2020 Apr 17]. N Engl J Med. 2020 Apr 17; NEJMc2009226.

13. Chen $\mathrm{N}$, Zhou M, Dong $\mathrm{X}$, et al. Epidemiological and clinical characteristics of $\mathbf{9 9}$ cases of $\mathbf{2 0 1 9}$ novel coronavirus pneumonia in Wuhan, China: a descriptive study. Lancet. 2020;395(10223):507-513.

14. Rasmussen SA, Smulian JC, Lednicky JA, Wen TS, Jamieson DJ. Coronavirus Disease 2019 (COVID-19) and pregnancy: what obstetricians need to know. Am J Obstet Gynecol. 2020 May; 222(5):415426.

15. Zhou $P$, Yang $X L$, Wang $X G$, et al. A pneumonia outbreak associated with a new coronavirus of probable bat origin. Nature. 579, 270-273 (2020).

16. Park WB, Kwon NJ, Choi SJ, et al. Virus isolation from the first patient with SARS-CoV-2 in Korea. J Korean Med Sci. 2020;35(7):e84.

17. Finlay BB, See RH, Brunham RC. Rapid response research to emerging infectious diseases: Lessons from SARS. Nat Rev Microbiol. 2004;2(7):602-7.

18. Hoffmann M, Kleine-Weber H, Schroeder S, et al. SARS-CoV-2 Cell Entry Depends on ACE2 and TMPRSS2 and Is Blocked by a Clinically Proven Protease Inhibitor. Cell. 2020;181(2):271-280.e8.

19. Wrapp D, Wang N, Corbett KS, et al. Cryo-EM structure of the 2019-nCoV spike in the prefusion conformation. Science. 2020;367(6483):1260-1263.

20. Matsuyama S, Taguchi F. Two-Step Conformational Changes in a Coronavirus Envelope Glycoprotein Mediated by Receptor Binding and Proteolysis. J Virol. 2009;83(21):11133-41.

21. Zoller M, Irlbeck M, Zwissler B. Coronavirus disease 2019. Saxena SK, editor. Vol. 69, Der Anaesthesist. Lucknow, India: Spring; 2020. 223-224 p.

22. Coutard B, Valle C, de Lamballerie X, Canard B, Seidah NG, Decroly E. The spike glycoprotein of the new coronavirus 2019-nCoV contains a furin-like cleavage site absent in CoV of the same clade. Antiviral Res. 2020;176:104742

23. Hendrickson CM, Matthay MA. Viral pathogens and acute lung injury: Investigations inspired by the SARS epidemic and the 2009 H1N1 influenza pandemic. Semin Respir Crit Care Med. 2013;34(4):475-86.

24. Li W, Moore MJ, Vasilieva N, et al. Angiotensin-converting enzyme $\mathbf{2}$ is a functional receptor for the SARS coronavirus. Nature. 2003;426(6965):450-454.

25. Ding $Y$, Wang $H$, Shen $H$, et al. The clinical pathology of severe acute respiratory syndrome (SARS): A report from China. J Pathol.
2003;200(3):282-9.

26. Levy A, Yagil Y, Bursztyn M, Barkalifa R, Scharf S, Yagil C. ACE2 expression and activity are enhanced during pregnancy. Am J Physiol Regul Integr Comp Physiol. 2008;295(6):R1953-R1961.

27. Brosnihan KB, Neves LA, Anton L, Joyner J, Valdes G, Merrill DC. Enhanced expression of Ang-(1-7) during pregnancy. Braz J Med Biol Res. 2004;37(8):1255-62.

28. Valdes G, Neves LA, Anton L, et al. Distribution of angiotensin-(1-7) and ACE2 in Human Placentas of Normal and Pathological Pregnancies. Placenta. 2006;27:200-207.

29. Delano MJ, Ward PA. The Immune System's Role in Sepsis Progression, Resolution and Long-Term Outcome. Immunol Rev. 2016 Nov; 274(1): 330-353.

30. Chen G, Wu D, Guo W, et al. Clinical and immunological features of severe and moderate coronavirus disease 2019.J Clin Invest. 2020;130(5):2620-9.

31. Pereira AC, De Jesús NR, Lage LV, Levy RA. Imunidade na gestação normal e na paciente com lúpus eritematoso sistêmico (LES). Rev Bras Reumatol. 2005;45(3):134-140.

32. Szekeres-Bartho J, Halasz M, Palkovics T. Progesterone in pregnancy; receptor-ligand interaction and signaling pathways. J Reprod Immunol. 2009;83(1-2):60-64.

33. Mor G, Aldo $P$, Alvero $A B$. The unique immunological and microbial aspects of pregnancy. Nat Rev Immunol. 2017;17(8):469-482.

34. Thaxton JE, Sharma S. Interleukin-10: A Multi-Faceted Agent of Pregnancy. Am J Reprod Immunol. 2010;63(6):482-491.

35. Peltier MR. Immunology of term and preterm labor. Reprod Biol Endocrinol. 2003;1:1-11.

36. Liu H, Wang LL, Zhao SJ, Kwak-Kim J, Mor G, Liao AH. Why are pregnant women susceptible to COVID-19? An immunological viewpoint. J Reprod Immunol. 2020;139:103122.

37. Racicot K, Mor G. Risks associated with viral infections during pregnancy. J Clin Invest. 2017;127(5):1591-9.

38. Wong SF, Chow KM, Leung TN, et al. Pregnancy and perinatal outcomes of women with severe acute respiratory syndrome. Am J Obstet Gynecol. 2004;191(1):292-297.

39. Chu H, Zhou J, Wong BHY, et al. Middle East Respiratory Syndrome Coronavirus Efficiently Infects Human Primary T Lymphocytes and Activates the Extrinsic and Intrinsic Apoptosis Pathways. J Infect Dis. 2016;213(6):904-914.

40. Wei SQ, Fraser W, Luo ZC. Inflammatory cytokines and spontaneous preterm birth in asymptomatic women: A systematic review. Obstet Gynecol. 2010;116(2):393-401.

Citation:

Pachnicki JPA, Wallim LR, de Moraes GV and de Lima SO. Maternal Physiological Changes in Pregnancyand Pathophysiology of COVID-19. Arch Physiol. 2021; 8:1. http://dx.doi.org/10.7243/2055-0898-8-1 